NASA/TM-2007-214822

\title{
Sensor Selection and Optimization for Health Assessment of Aerospace Systems
}

William A. Maul

Analex Corporation, Cleveland, Ohio

George Kopasakis

Glenn Research Center, Cleveland, Ohio

Louis M. Santi

Jacobs ESTS Group, Huntsville, Alabama

Thomas S. Sowers and Amy Chicatelli

Analex Corporation, Cleveland, Ohio

July 2007 


\section{NASA STI Program . . . in Profile}

Since its founding, NASA has been dedicated to the advancement of aeronautics and space science. The NASA Scientific and Technical Information (STI) program plays a key part in helping NASA maintain this important role.

The NASA STI Program operates under the auspices of the Agency Chief Information Officer. It collects, organizes, provides for archiving, and disseminates NASA's STI. The NASA STI program provides access to the NASA Aeronautics and Space Database and its public interface, the NASA Technical Reports Server, thus providing one of the largest collections of aeronautical and space science STI in the world. Results are published in both non-NASA channels and by NASA in the NASA STI Report Series, which includes the following report types:

- TECHNICAL PUBLICATION. Reports of completed research or a major significant phase of research that present the results of NASA programs and include extensive data or theoretical analysis. Includes compilations of significant scientific and technical data and information deemed to be of continuing reference value. NASA counterpart of peer-reviewed formal professional papers but has less stringent limitations on manuscript length and extent of graphic presentations.

- TECHNICAL MEMORANDUM. Scientific and technical findings that are preliminary or of specialized interest, e.g., quick release reports, working papers, and bibliographies that contain minimal annotation. Does not contain extensive analysis.

- CONTRACTOR REPORT. Scientific and technical findings by NASA-sponsored contractors and grantees.
- CONFERENCE PUBLICATION. Collected papers from scientific and technical conferences, symposia, seminars, or other meetings sponsored or cosponsored by NASA.

- SPECIAL PUBLICATION. Scientific, technical, or historical information from NASA programs, projects, and missions, often concerned with subjects having substantial public interest.

- TECHNICAL TRANSLATION. Englishlanguage translations of foreign scientific and technical material pertinent to NASA's mission.

Specialized services also include creating custom thesauri, building customized databases, organizing and publishing research results.

For more information about the NASA STI program, see the following:

- Access the NASA STI program home page at http://www.sti.nasa.gov

- E-mail your question via the Internet to help@sti.nasa.gov

- Fax your question to the NASA STI Help Desk at 301-621-0134

- Telephone the NASA STI Help Desk at 301-621-0390

- Write to: NASA Center for AeroSpace Information (CASI) 7115 Standard Drive Hanover, MD 21076-1320 
NASA/TM-2007-214822

AIAA-2007-2849

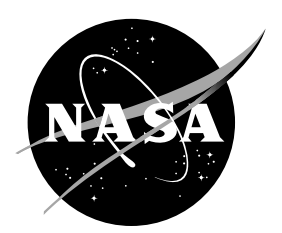

\section{Sensor Selection and Optimization for Health Assessment of Aerospace Systems}

William A. Maul

Analex Corporation, Cleveland, Ohio

George Kopasakis

Glenn Research Center, Cleveland, Ohio

Louis M. Santi

Jacobs ESTS Group, Huntsville, Alabama

Thomas S. Sowers and Amy Chicatelli

Analex Corporation, Cleveland, Ohio

Prepared for the

Infotech at Aerospace 2007 Conference and Exhibit

sponsored by the American Institute of Aeronautics and Astronautics

Rohnert Park, California, May 7-10, 2007

National Aeronautics and

Space Administration

Glenn Research Center

Cleveland, Ohio 44135 


\section{Acknowledgments}

The authors gratefully acknowledge the Integrated System Health Management Project under the NASA Exploration Technology Development Program and the Integrated Vehicle Health Management Project under the NASA Aviation Safety Program for their interest in and support of this effort.

Level of Review: This material has been technically reviewed by technical management.

Available from

NASA Center for Aerospace Information 7115 Standard Drive

Hanover, MD 21076-1320
National Technical Information Service 5285 Port Royal Road Springfield, VA 22161 


\title{
Sensor Selection and Optimization for Health Assessment of Aerospace Systems
}

\author{
William A. Maul \\ Analex Corporation \\ Brook Park, Ohio 44142 \\ George Kopasakis \\ National Aeronautics and Space Administration \\ Glenn Research Center \\ Cleveland, Ohio 44135 \\ Louis M. Santi \\ Jacobs ESTS Group \\ Huntsville, Alabama 35812 \\ Thomas S. Sowers and Amy Chicatelli \\ Analex Corporation \\ Brook Park, Ohio 44142
}

\begin{abstract}
Aerospace systems are developed similarly to other large-scale systems through a series of reviews, where designs are modified as system requirements are refined. For space-based systems few are built and placed into service-these research vehicles have limited historical experience to draw from and formidable reliability and safety requirements, due to the remote and severe environment of space. Aeronautical systems have similar reliability and safety requirements, and while these systems may have historical information to access, commercial and military systems require longevity under a range of operational conditions and applied loads. Historically, the design of aerospace systems, particularly the selection of sensors, is based on the requirements for control and performance rather than on health assessment needs. Furthermore, the safety and reliability requirements are met through sensor suite augmentation in an ad hoc, heuristic manner, rather than any systematic approach. A review of the current sensor selection practice within and outside of the aerospace community was conducted and a sensor selection architecture is proposed that will provide a justifiable, defendable sensor suite to address system health assessment requirements.
\end{abstract}

\section{Introduction}

Aerospace systems are developed similarly to other large-scale systems through a series of design reviews. At each design stage, decisions and trades are made about all aspects of the vehicle: hardware, software, functionality, operations, etc. Each component, including sensors, must "buy its way onboard." The costs and risks associated with flight dictate that unnecessary components be filtered from the design; therefore, all components must justify their existence and purpose onboard the vehicle. To achieve this, a component must satisfy a quantifiable and verifiable set of requirements that will demonstrate its benefit and purpose to the system. In addition, any risk to the system must also be identified.

Traditionally, in flight systems, sensors are primarily selected through an ad hoc heuristic process, where various domain groups are polled as to what sensors they require in the system. Although safety and reliability are just as important as system performance, the sensors are primarily selected based on control requirements and performance assessment, rather than on health monitoring and management. To be incorporated into the design process, accepted methodologies and procedures must be established that provide justification of the selected sensors within the constraints of the vehicle requirements. To precisely quantify the benefits of sensors in flight systems, heuristic sensor selection approaches must give way to systematic techniques that are based on accepted selection criteria and performance metrics that measure their value to the system.

Therefore, substantial benefit to the design and development process can be realized by utilizing a sensor selection methodology, specifically directed toward system health assessment. The purpose of this study is to review the current state of sensor selection within and outside of the aerospace system community and to propose a sensor 
selection architecture that will provide justification of sensor inclusion based on the net benefit from the health assessment perspective. This review is not intended to be encompassing in terms of directly comparing the various techniques against each other and assessing their performance. Rather, the review is based on categorizing salient features of these approaches and the desirability of certain features when they are applied to aerospace flight systems.

The paper is organized as follows. Sections II and III will review and discuss various techniques used historically for sensor selection. Section IV will discuss in detail the proposed sensor selection architecture originally designed to address health assessment for propulsion systems. Finally we will conclude with a discussion of proposed research areas that will advance the capabilities and credibility of the sensor selection process for aerospace diagnostic applications. A design example will be included in the appendix that will demonstrate an application of the proposed sensor selection technique.

\section{An Overview of the Sensor Selection Process}

A number of sensor selection approaches, applied to a variety of systems and for a variety of purposes, have been developed over the years. For the methods described in this review, the sensor selection process can be divided into two parts: an evaluation module and an optimization module. The evaluation module generates a performance metric for the sensor suite or network under review, and the optimization module searches through the space of all possible sensor suites and selects additional suites for further evaluation. The process is continued until the "best" or the optimum sensor suite is obtained.

\section{A. Sensor Selection Evaluation}

The goal of the sensor selection process is to provide a suite of sensors that fulfill specified performance requirements within a set of system constraints. These performance requirements are defined as the Figures of Merit (FOMs) of the system, and considerable research has focused on how to represent these FOMs in algorithmic form. The applied set of FOMs should reflect the overall goal of the selection process. The following list of general FOM categories was selected after reviewing the available literature and research.

- Observability - This category considers how well the sensor suite will provide information about the given system process, which parameters that are directly observed, and which parameters can be inferred.

- Sensor Reliability/Sensor Fault Robustness-This category addresses sensor reliabilities and how sensor availability impacts the overall sensor suite performance.

- Fault Detectability/Fault Discriminability-This category specifically addresses whether the sensor suite can detect and discriminate system failures.

- Cost-This category can include development, purchase, and maintenance costs for the sensors as well as resource and communication costs.

These FOMs will be discussed in greater detail in section III, where we will identify specific methodologies and highlight examples of research that define them.

\section{B. Optimization Processes}

Analysis has shown that general sensor selection problems addressing diagnosability, or observability, are NPcomplete and are therefore computationally intractable (ref. 1). These types of problems require fast approximate search solutions in order to generate acceptable results in reasonable time. Where possible, Brute Force or Exhaustive Search methods are preferable, but they examine all possible candidates and as systems become more complex, the time involved grows prohibitive. Therefore, methods have been developed which attempt to refine the searches through determination of potential candidates in order to find the optimal or near-optimal solution. Regardless of the selection strategy, sensor selection is based on a set of criteria called the objective function which is an algorithmic representation of established FOMs and system constraints.

Many techniques have been developed for general optimized solution searches. These techniques range from applying constraints on the objective function to streamline the optimization process (ref. 2) to applying advanced artificial analysis techniques such as genetic algorithms and simulated annealing algorithms (ref. 3). Unique optimization techniques have been proposed such as particle swarm optimization (ref. 4) and cutting and surrogate constraint analysis (ref. 5). The point of this section is not to review each search solution or optimization technique but to identify the variety of algorithms available. Optimization techniques are well documented (ref. 6). Table 1 reports the algorithms encountered in this literature review and references that utilized them. 
TABLE 1.- SENSOR SELECTION OPTIMIZATION TECHNIQUES ENCOUNTERED AND ASSOCIATED REFERENCES

\begin{tabular}{|l|l|}
\hline \multicolumn{1}{|c|}{ Optimization technique } & \multicolumn{1}{c|}{ Researcher } \\
\hline Constraint-Based Search & Narasimhan (ref. 7), Mushini (ref. 8) \\
\hline Exhaustive/Brute Force Search & $\begin{array}{l}\text { Debouk (ref. 2), Mushini (ref. 8), Narasimhan (ref. 7), Madron } \\
\text { (ref. 9), Worden (ref. 3) }\end{array}$ \\
\hline Genetic Algorithms & $\begin{array}{l}\text { Musulin (ref. 10), Mushini (ref. 8), Spanache (ref. 11), Sen } \\
\text { (ref. 12), Santi (ref. 13), Worden (ref. 3) }\end{array}$ \\
\hline Particle Swarm Optimization & Zhang (ref. 14) \\
\hline Graph Theory-Spanning Trees and Cutsets & Ali (refs. 15 and 16), Bagajewicz (refs. 17 and 18) \\
\hline Cutting and Surrogate Constraint Analysis & Azam (ref. 19) \\
\hline Mixed Linear Integer Programming & Bagajewicz (refs. 20, 21, and 22), Chmielewski (ref. 23) \\
\hline Simulated Annealing Algorithm & Worden (ref. 3) \\
\hline
\end{tabular}

\section{Historical Review of Sensor Selection Processes}

The background for the selection of the FOMs presented in section II is described below. These metrics are based on qualitative as well as quantitative methods and derived from a number of disciplines such as controls theory, estimation theory, data reliability and failure analysis. The subsequent subsections will review some of the research development that has been done in each of the first three categories: observability, sensor reliability/sensor fault robustness and fault detectability/fault discrimination. The fourth category, cost, is a general penalty-driven FOM grouping and is very straightforward conceptually and in implementation. There is overlap between the arbitrarily defined categories, and the final sensor selection FOM may be a combination of performance and cost metrics from any or all of them.

\section{A. Observability}

Of ultimate importance for any sensor suite is the concept of observability. Basically, observability is the capacity of the sensor network to provide information about the state parameters deemed important for performance monitoring, health assessment, and/or control of the system. This information may be provided as direct measurements of the system parameters or as in data reconciliation, the reconstruction of unobservable system parameters based upon observable or sensed ones. Approaches vary in the determination of the observability of the sensor network. Some treat observability as a true or false property, while others devised more sophisticated schemes to display the degree of observability.

Some approaches used graph-based analysis for which the structural information of a system is represented by a directed graph, called a digraph. Observability is then based on analysis of cutsets and cycles generated from the digraph. Kretsovalis (ref. 24) proposed two graphically-based algorithms for classification of observable and redundant variables. Luong (ref. 25) also used graph-based analysis to establish an incidence matrix, relating the process relationships to the state variables qualitatively. Decomposition of this incidence matrix using a GaussJordan elimination process identified whether an unmeasured variable was observable and whether a measured variable was redundant. Using graph theory and cutsets, Bagajewicz (ref. 18) defined the degree of observability and degree of redundancy for a sensor network. For an unmeasured variable, the degree of observability is the maximum number of sensors that can be eliminated with the variable still observable; for measured variables, the degree of redundancy is the maximum number of sensors that can be eliminated and the measurement remains redundant. These concepts were combined to define a degree to which system variables can be estimated by the measurement network.

Other approaches attempted to define the degree of observability by analyzing the numerical relationships of the system process. By taking advantage of linear state space system theory, observability and controllability can be represented in matrix form. The system is observable or controllable if the observability or controllability matrices are of full rank or nonsingular. These represent binary conditions as to whether the system is observable/controllable or not. Dochain (ref. 26) considered both the rank and condition number of the observability matrix for an optimal sensor placement within a bioreactor system. The Gramian of both the observability and controllability matrices have been used by researchers (refs. 27 and 28) to develop scalar metrics that indicated the degree of each respective property.

A large amount of research has been devoted to control design and actuator/sensor placement locations, particularly in structural-type problems. Papadopoulos (ref. 29) investigated a number of quantitative techniques used to optimize structural sensor placement to select sensor locations that would observe the maximum amount of system information, in this case energy. Each technique attempted to characterize the amount of energy the sensors 
would observe and eliminate potential sensor locations that had minimal observability. Hac (ref. 30) proposed a methodology that incorporated eigenvalues from both the observability and controllability matrices to select sensor locations for control of flexible structures.

Several researchers used the predictive error of a given sensor suite relative to a specific reconciliation technique as the performance metric. After performing matrix decomposition on the linear system model coefficients, Madron (ref. 9) proposed a simple local optimization of this baseline set of sensors by computing the prediction precision of unmeasured required state parameters. The baseline sensor suite performance was compared to suite variations where a single sensor was removed and the objective function recomputed. Chmielewski (ref. 23) developed the framework for the justification and incorporation of the error covariance matrices for sensor suite performance properties and demonstrated how that could be optimized with other suite performance constraints using mixed linear integer programming techniques. Musulin (ref. 10) proposed a sensor selection process that maximized a Kalman Filter performance, via the error covariance matrix diagonal terms. Musulin pointed out that although the objective is to maximize the performance of the filter for all variables, maximizing the performance for one particular variable may not be compatible with maximizing it for others, resulting in a conflict. Therefore they proposed two possible approaches; one method would select the variable with the lowest performance value. The second approach would evaluate the performance of each variable relative to an "ideal" sensor suite performance, combining into an overall performance metric. Mushini (ref. 8) used a similar approach of maximizing a Kalman Filter performance for an aircraft gas turbine engine. The metric was defined as the summation of the error covariance matrix diagonal terms normalized to the reference or ideal system performance.

\section{B. Sensor Reliability/Sensor Fault Robustness}

Regardless of the purpose of the sensor network, consideration must be made for the availability of the sensor signal information when it is required. Sensor faults can be common, and the potential for interruption of information flow and how that is to be handled is an important consideration in the sensor network design analysis.

Luong (ref. 25) investigated sensor network reliability from a control perspective that the reliability of an instrumentation system is the probability that information required for control are available through measurements or deduction during some time period. Based on that idea, an overall sensor network reliability metric was defined as an expression of the individual sensor reliabilities. Sensor reliabilities were expressed as a function of time; therefore, the integration of the time profile for each sensor network was used as a quantitative comparison metric between competing networks.

Ali (ref. 15) introduced the concept of reliability of the estimation of variables, which relates the sensor reliability with its availability to provide system state estimations. The focus of the research was for a given sensor network, how many different ways can a variable be estimated and if sensors fail, can a variable still be estimated. The network objective function was defined as the minimum product of the input sensor reliabilities for each estimated parameter. The initial processing routine involved graph theory concepts of chords, cutsets, and spanning trees to establish sets of estimated variables and their associated sensors used to observe the variable. This objective function was extended to include hardware redundancy (measuring a variable using more than one sensor) and spatial redundancy (more than one way of estimating a variable) (ref. 16). Within the graph theory algorithm, the sensor network can be optimized with respect to only a single criterion; therefore, this objective function was adapted for processing with genetic algorithms (ref. 12) and mixed-integer nonlinear program (MINLP) (ref. 20) which enabled it to be evaluated with other performance metrics, such as sensor costs.

Another consideration of the sensor network is the performance in the presence of sensor faults. Sensor networks must be robust to sensor faults, both in being able to perform adequately with a faulty sensor within the network and being able to continue performance adequately if a sensor is removed. Bagajewicz (ref. 17) proposed a set of performance metrics for a sensor suite that defined the ability of the sensor network to detect sensor faults (error detectability) and handle sensor faults relative to reconciliation precision performance (availability — precision after failed sensor removed, and resilience-precision in the presence of a sensor fault). Bagajewicz (ref. 21) extended this research from the graphical tree-search-solution procedure to the explicit MINLP formulation and also extended the theory for explicit consideration of hardware redundancy.

\section{Fault Detectability/Fault Discriminability}

For fault diagnosis, the ability of the sensor network to detect and discriminate failure modes and anomalous conditions is of prime importance. This is an extension of the observability analysis where specific state variables or groups of state variables and their response are indicative of health conditions. The performance metric must define the sensor network's ability to distinguish these fault conditions from nominal operation (fault detection) and distinguish these fault conditions from each other (fault discrimination). 
Significant research focuses on the qualitative or heuristic representation of the fault progression process. Research conducted by Raghuraj (ref. 31), Bhushan (ref. 32), and Bagajewicz (ref. 22) utilized directed or signdirected graphs to generate fault signatures. A directed graph of a hypothetical process with faults, $\mathrm{F}_{\mathrm{i}}$, connecting to sensors, $S_{i}$, indicating that the fault will affect the reading of the corresponding sensor, is displayed in figure 1 . These signatures provided only an indication that the sensor should respond to the particular fault condition, but the actual signature, magnitude or rate, was not utilized. Spanache (ref. 11) used the physical constraints of the system to develop a set of analytical redundancy relationships between the measured and unmeasured variables and qualitatively established an influence matrix between system components and possible sensor parameters. Fault signatures were assigned to a failed component without regard to specific component failure modes, and hence a fault signature matrix was generated between the component faults and the influenced analytical redundancy relationships. Narasimhan (ref. 7) used temporal causal graphs, and Yan (ref. 33) used a CAD system model to develop qualitative fault signatures. Representing the fault signature response qualitatively avoids the cost of simulating the fault in hardware and/or software and often this is the only level of fault information available during the early development of a system.

In an effort to incorporate more fault information, Zhang (ref. 14) proposed a quantified directed graph (QDG) to model the fault propagation. For the QDG, each node represented a potential sensor with an associated signal-tonoise ratio, and each arc contained the fault propagation gain and direction between the sensors, along with fault propagation time. A sensor detectability value was computed for each sensor in the suite for a predefined set of faults. Fault detectability was averaged over the available sensor suite and evaluated against an assigned lower bound. Fault resolution was still treated somewhat qualitatively by comparing sets of sensors influenced by each fault.

Azam (ref. 19) proposed a method that used a system model and reliability data to establish cause/effect dependencies between the faults of interest and the effects of faults on observable system parameters. The detection and false alarm probabilities associated with the failure source and observable discrepancy were utilized. A general multiple fault diagnosis algorithm was used to search for the most likely candidate fault that explained the set of observed discrepancies. Three diagnostic performance metrics were established for each potential sensor based on the decision probability error from a number of simulation runs. Selection evaluation was performed for candidate sensor networks by summing these performance metrics over the sensor suite and incorporating cost constraints.

Santi (ref. 13) utilized a high-fidelity dynamic model of a rocket propulsion system to generate a simplified linear model. Health parameters were identified within the linear model that represented specific fault conditions. This research focused on mean-shift faults in the system. An inverse diagnostic model was developed which predicted the change in health parameters based on sensor inputs. Fault trajectories were generated and metrics were established for detection timeliness and fault discrimination. Fault detection required sufficient measurement deviation to discriminate a fault from normal operation variance. Detection thresholds were established based on the historical information of measurement variance, anticipated process variance and defined false alarm constraints of the system. Figure 2 illustrates a two-dimensional representation of the fault trajectory space where the axes represent sensor change from a nominal state (the origin) in increments of the sensor nominal standard deviation $(\sigma)$. The diagnostic performance metrics were based on the distance measured between fault trajectories for fault discrimination and inferred time to detection threshold areas for detection timeliness. In addition, fault risk reduction measures were incorporated into the evaluation process to allow weighting of individual failure modes based on criticality and occurrence rates. 


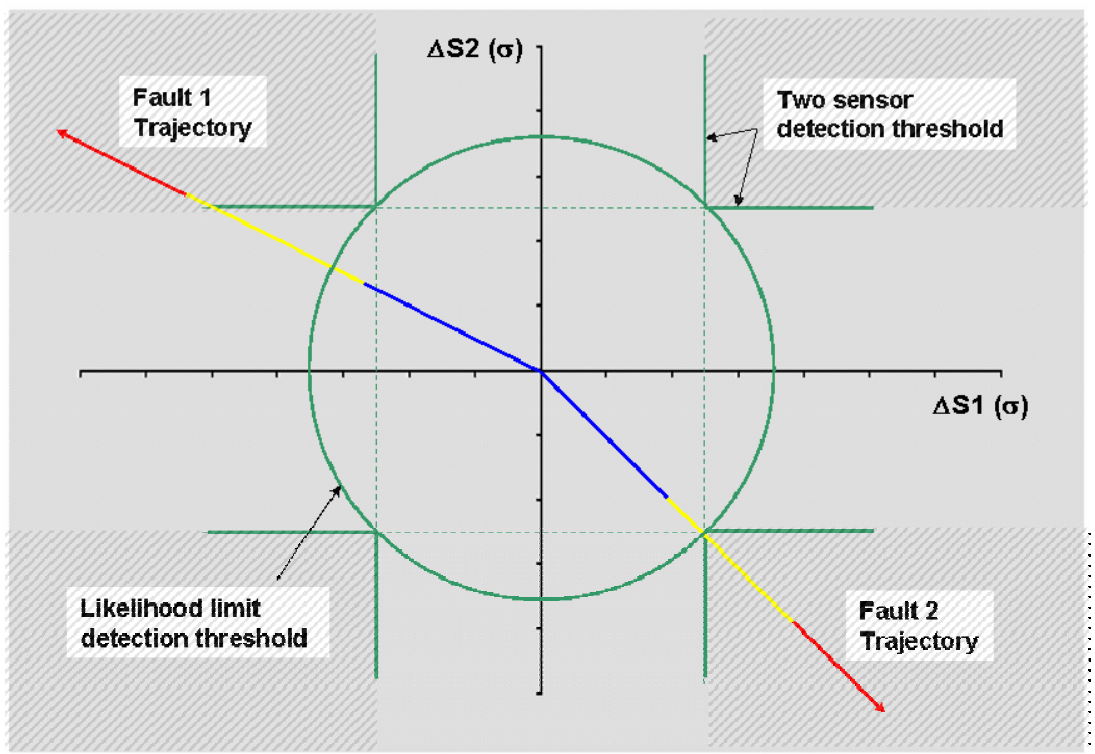

Figure 2.-Nondimensional fault trajectory space for two sensors, S1 and S2.

\section{Proposed Sensor Selection Approach}

While the design of aerospace systems/subsystems does not necessarily require an analytical methodology to select the location and quantity of sensors, sensor placements based solely on expert "best guess" can result in nonuniformity across the system and can be unverifiable especially for competing objectives such as maximizing safety and reducing costs. Therefore, a systematic approach based on accepted techniques and metrics is desired that allows for sensor network trades and consistent evaluation of metrics across the system. We direct our focus toward ensuring that the health assessment requirements are met by the sensor network; in that respect, several key questions must be addressed through evaluation of the sensor networks:

- Can the network provide a sufficient level of detectability for the failure modes of concern? Is the sensor network able to detect each failure and how early after the fault is initiated?

- Can the network discriminate between the various system failure modes? Failure discrimination need only be performed to the level where remediation strategies vary.

- Can the network perform the diagnosability within the cost constraints established by the systems? This would include development, installation and maintenance costs, processing costs, power requirements, and weight.

- How robust is the network in the presence of sensor failures? What sensors require redundancy? Are there common mode failures among the sensor network that could be overcome?

In addition, it is important to utilize the maximum level of fidelity available in establishing the justification for sensor selection; therefore, quantitative approaches are preferred over qualitative approaches, but the methodology should be flexible enough to incorporate the best system design information available. The methodology selected should also focus on known failure modes and allow the user to incorporate weighting factors that represent both fault criticality and sensor costs. Finally, the methodology should incorporate other metrics, such as sensor reliability and sensor fault robustness, as needed.

The approach that we have selected will advance the method described in reference 13, Systematic Sensor Selection Strategy (S4). This approach to sensor selection provides an architecture that fulfills the objectives outlined above, and a specific application of the S4 methodology to a propulsion system is provided in the appendix. Although the application utilizes a specific diagnostic engine, this methodology may be applicable to a wide range of diagnostic techniques. In addition, the architecture of this methodology should allow the implementation of other evaluation metrics and optimization processes like those reviewed in sections II and III. The overall objectives and architecture for the proposed sensor selection approach are given below. 


\section{A. Objectives}

The goal of the diagnostic sensor selection methodology is to provide a systematic, justifiable, creditable evaluation of the available sensor suite, relative to the diagnostic requirements. The methodology should be applicable to multiple types of systems: propulsion, electrical, hydraulic, etc. Each application and implementation may require unique metric algorithms, application-specific diagnostic models, and tailored optimization selection strategies, therefore the systematic approach should be flexible and modular.

The methodology should be applicable across various phases of operation for the system being applied. Often the same system may have distinct modes of operation where diagnostic requirements may indicate unique sensor networks. For example, the faults and corresponding sensors required to monitor, detect, or diagnose those faults at one operating mode of the system (e.g., engine firing) may not be the same required at another (e.g., quiescence or dormant checkout phase).

The methodology should apply throughout the design phase, regardless of the fidelity of simulations and domain information. As noted by both Santi (ref. 13) and Musulin (ref. 10), the fidelity of the model utilized will impact the performance, health assessment, or data reconciliation, and therefore the sensor suite selection process. Chmielewski (ref. 23) proposed that the quality of the reconciled values will depend more on the sensor configuration than the particular reconciliation algorithm employed. Therefore, the inputs, simulations, and fault and sensor information, based on "best available information" are required at each stage of the design process. Either the design analysis tool should demonstrate applicability throughout or define where in the process it is relevant.

Finally, the sensor network performance depends upon both the sensor suite available and the analysis technique applied. An optimum sensor network established for a specific technique may not be appropriate for another technique. Care must be taken in generalizing the performance results across analysis functions to ensure appropriate solutions. The sensor selection architecture should allow different diagnostic assessment modules, optimization techniques, and performance metrics to be utilized.

To that end we are proposing an architecture that will enable the following capabilities:

- A toolbox of representative cost and performance metrics, allowing the user to specify how those metrics will be defined and combined.

- Weighting capability at the component fault, sensor measurement, and performance criteria metric levels to allow for importance to be established by the user.

- Established interfaces between modules to allow implementation of diagnostic modules and optimization techniques. A plethora of diagnostic algorithms, optimization search algorithms, and system simulations exist that could be utilized for this methodology. Standardization and definitions of interfaces within the framework will enable the inclusion of these other modules.

\section{B. Architecture}

Figure 3 illustrates the sensor selection architecture proposed by Santi (ref. 13) and the one that the authors have selected as the framework for further development and verification. The architecture can be segmented into three groups: Knowledge Base, Iterative Down-Select Process, and Final Selection.

Each segment of the architecture is summarized as follows:

- Knowledge Base-Information about the host system is gathered. If data on the host system is not available, experience from similar systems can provide a basis from which to define and collect pertinent health assessment information. The knowledge base includes the health-related information and the system simulation.

- Health-Related Information-This information is extracted from domain experts, manufacturer reports, Failure Modes and Affects Analysis (FMEA), and hazard analysis studies and will include fault signatures, fault progressions, component/sensor reliabilities and sensor characteristics (e.g., noise). This data is used as inputs to the system diagnostic model, sensor suite merit algorithm, and downselect algorithm. In addition, it can also provide information to the system simulation.

- System Simulation-Provides input in the form of data sets for the system diagnostic model and statistical evaluation algorithm. This module may be a collection of simulations of varying fidelities and applicable at varying stages of the system operation. When available and appropriate, real test or flight data could be provided from this module. 


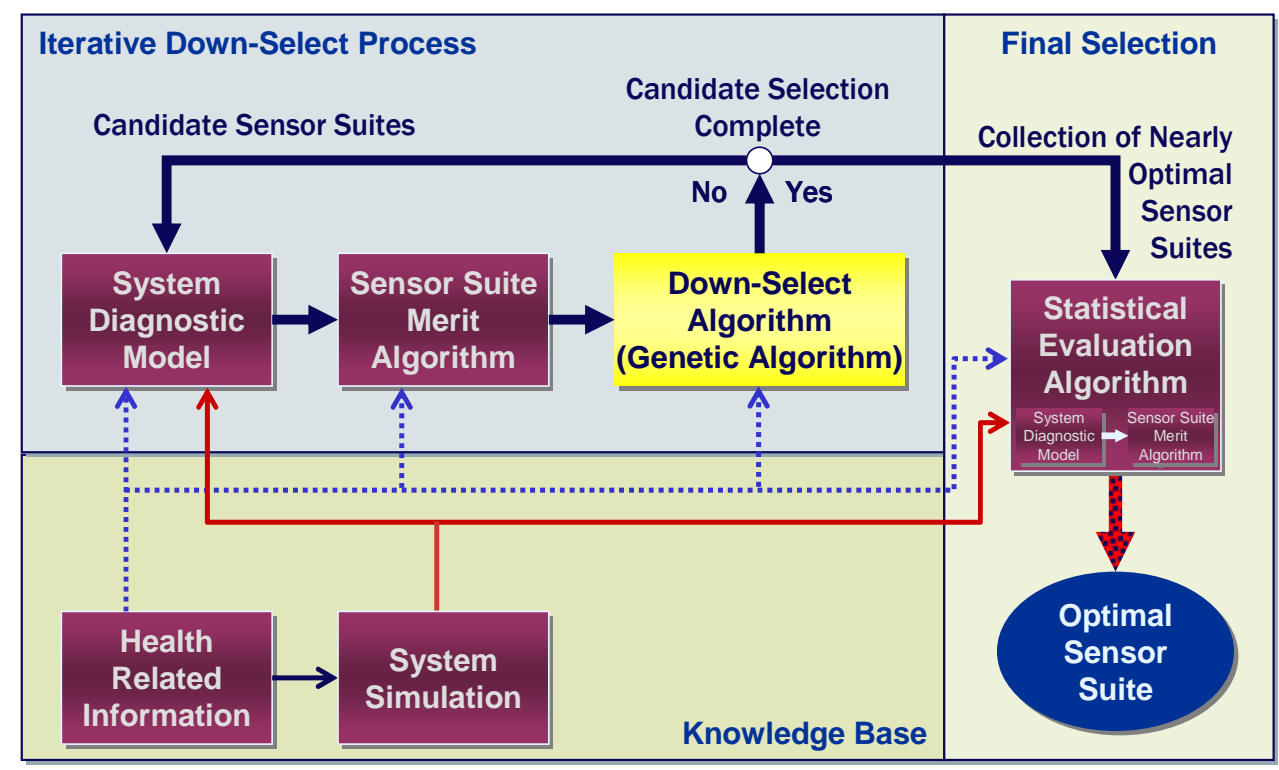

Application Specific

Figure 3.-Proposed diagnostic sensor selection architecture.

- Iterative Down-Select Process-An iterative procedure to select a group of near-optimal sensor suites for health assessment. The process involves sequentially generating and evaluating a collection of candidate sensor suites, eventually converging towards a more optimum sensor suite based on the defined performance metric or objective function. The procedure includes a system diagnostic model, a sensor suite merit algorithm, and a down-select algorithm.

- System Diagnostic Model - The performance of the diagnostic system is measured for each candidate sensor suite at each relevant operational mode. The measures of performance are provided to the sensor suite merit algorithm. A key feature for this model is that it must be complete and capable of providing diagnostic analysis for all or any subset of the possible sensors. A diagnostic model that must be redefined for each new sensor suite would not be appropriate for this architecture. It receives input primarily from the down-select module and outputs information to the sensor suite merit module.

- Sensor Suite Merit Algorithm-The merit algorithm provides a score for each candidate sensor suite. It generates quantified metrics utilizing performance information from the system diagnostic model along with other pertinent characteristics of the measurement suite being evaluated. It receives input from the system diagnostic model and it outputs information to the down-select algorithm.

- Down-Select Algorithm-The down-select algorithm is a search algorithm that utilizes an optimization technique employed to select sensor suites that will allow progression toward an optimal or nearoptimal solution. Due to the large search space of the candidate sensor suites, the use of an optimized search algorithm, such as a genetic algorithm, allows for a timely generation of candidate sensor suites. The down-select algorithm receives inputs from the health-related information and sensor suite merit algorithm, and generates a collection of new candidate sensor suites that should "evolve" toward the optimum solution.

- Statistical Evaluation Algorithm-The statistical evaluation algorithm is intended as a final robustness test of each candidate sensor suite. When constructing the iterative loop of the down-select process, often there is a trade between fidelity and speed. Therefore, a more rigorous evaluation is employed when a finite collection of "optimum" measurement sets has been determined. The statistical evaluation algorithm replicates portions of the down-select process but with additional uncertainty effects such as sensor and system noise characteristics, and variations in fault dynamics. Each sensor suite is evaluated by the system diagnostic model and the sensor suite merit algorithm. The final sensor suites can be evaluated on the performance with these higher fidelity simulations. The statistical evaluation algorithm uses input from the health-related information, system simulation data, and the collection of nearly optimal sensor suites. 
The result of this selection process is a set of viable candidates that the designer can further assess and carry forward in the system design process. Each suite will have metrics measured against its performance to enable direct comparisons. This approach can demonstrate the impact of individual sensor measurements or to attempt to quantify relative benefit between sensor networks. Trade studies can be conducted to compare optional sensor networks and identify health monitoring gaps. Most importantly, the sensor network designer will have a valuable tool with which he can defend the selection made.

\section{Concluding Remarks}

The sensor selection process is the evaluation of sensor networks with the objective of fulfilling specified performance requirements within a set of system constraints. For the health assessment of aerospace systems, the performance requirements include fault detection, fault resolution, and a timely diagnosis. Other metrics can include gross error detectability and the ability to perform in the event of a sensor fault, as well as minimizing sensor costs. The paper includes a review of current literature from other disciplines (e.g., industrial and chemical processing applications) in order to assess the state of sensor selection technologies that might be applicable to aerospace systems. This investigation offers a prelude to the proposed architecture based on what is considered to be important criteria for a sensor selection approach for aerospace applications. A sensor selection architecture was proposed that will expand on the research conducted by Santi (ref. 13). The architecture's flexibility will enable broader combinations of evaluation metrics to be incorporated. Further development and demonstration is required to advance the confidence of this approach with aerospace design applications. In addition, applicability to other systems beyond propulsion elements needs to be established. This will require the definition of a formal architecture and interfaces that will allow other modules to be easily integrated. Demonstrations need to be conducted to determine applicability in other areas. 



\section{Appendix-Systematic Sensor Selection Strategy (S4) Example}

\section{A. Nomenclature}

$\begin{array}{ll}C_{j} & \begin{array}{l}\text { fault criticality weighting factor } \\ \text { detection distance metric for each training fault case } \\ D_{\text {resid }}\end{array} \\ D_{\text {Total }} & \begin{array}{l}\text { detection distance metric for the tested fault case } \\ \text { normalizing term for the penalty function }\end{array} \\ K_{\mathrm{N}} & \text { number of fault cases evaluated } \\ N_{\mathrm{FC}} & \text { number of tested fault cases } \\ N_{\mathrm{FM}} & \text { number of sensors in evaluated suite } \\ N_{\mathrm{S}} & \text { actual number of sensors in candidate sensor suite } \\ N_{\text {Sactual }} & \text { desired number of sensors in solution sensor suite } \\ N_{\mathrm{Sdesired}} & \text { probability of the correct fault detection } \\ P_{\mathrm{FD}} & \text { penalty term component of the merit function } \\ P_{\mathrm{PEN}} & \text { utility cost term applied to each sensor in the current suite } \\ U_{i} & \text { total utility cost associated to the current sensor suite } \\ U_{S} & \text { individual fault type weighting factor } \\ W_{j} & \text { weight of the penalty term } \\ W_{\text {penalty }} & \text { recovered normalized residual sensor value } \\ Y_{R} & \text { detection threshold } \\ Y_{T} & \end{array}$

Following the methodology and procedure outlined in figure 3, S4 was applied to subsystem components of the Space Shuttle Main Engine (SSME). While sensor selection methodologies are typically applied at the system level, the scope of this example was limited to facilitate presentation. More detail on this example problem along with entire S4 methodology is presented in the S4 User and Software Guide (ref. 34).

As displayed in the simplified flow schematic of the SSME in figure A1, there are 3 targeted fault conditions and 10 candidate sensors in this example. The three fault conditions of interest in this example are a nozzle coolant flow leak, a high pressure oxidizer turbopump efficiency loss, and an oxidizer preburner fuel injector resistance increase. The candidate sensors are defined in table A1 and are the only sensors considered in this exercise.

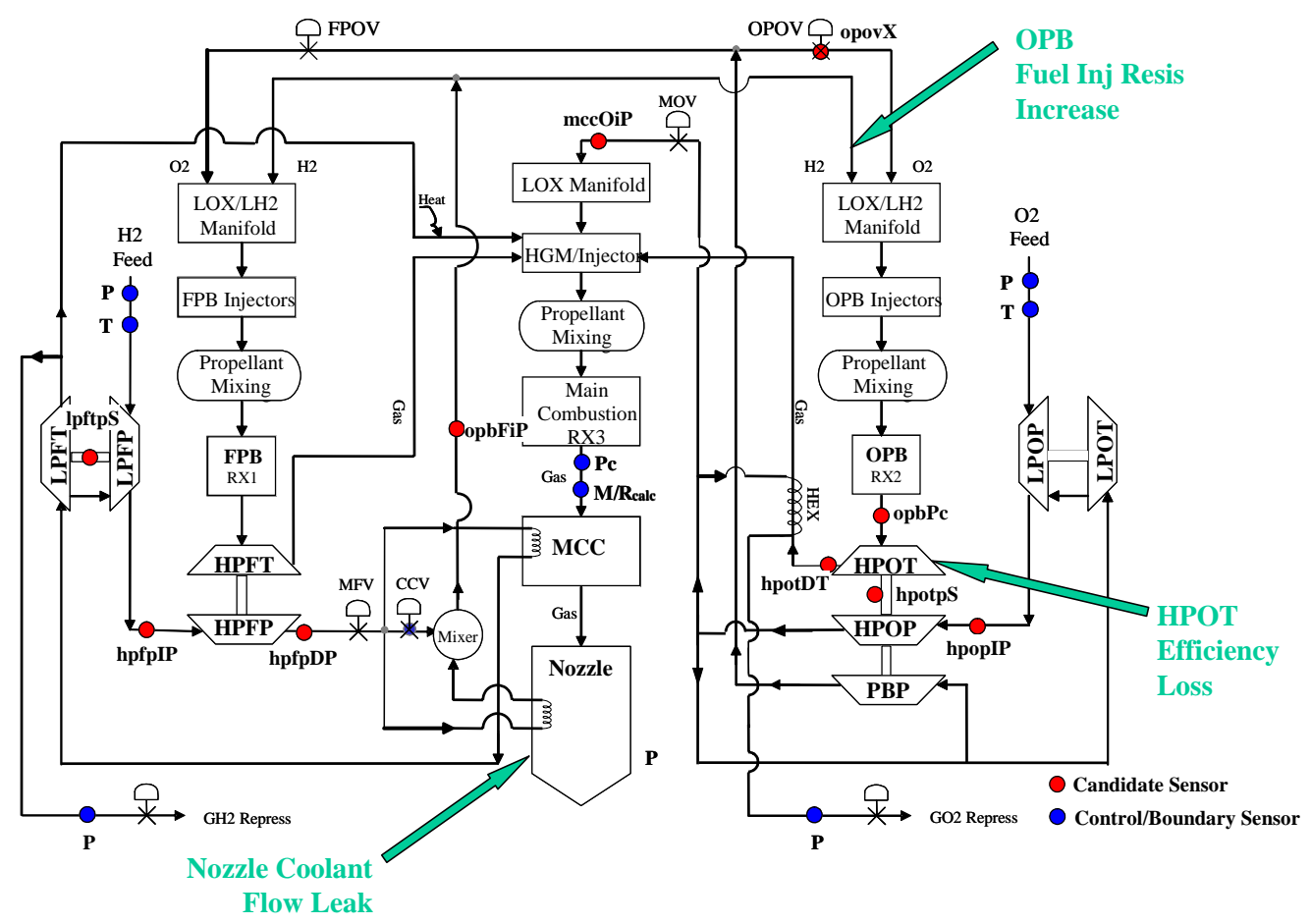

Figure A1.-SSME flow path schematic. 
TABLE A1.-CANDIDATE SENSORS

\begin{tabular}{|l|l|}
\hline \multicolumn{1}{|c|}{ Simulation variable } & \multicolumn{1}{c|}{ Sensor description } \\
\hline hpfpDP & HPFP discharge pressure \\
\hline hpfpIP & HPFP inlet pressure \\
\hline lpftpS & LPFTP speed \\
\hline hpopIP & HPOP inlet pressure \\
\hline hpotpS & HPOTP speed \\
\hline opbPc & OPB chamber pressure \\
\hline opovX & OPOV actuator position \\
\hline mccOiP & MCC OX injector pressure \\
\hline opbFiP & OPB fuel supply duct inlet pressure \\
\hline hpotDT & HPOT discharge temperature \\
\hline
\end{tabular}

The SSME Power Balance Model was used as the simulation model because it is well established. Three simulation variables were identified, each representing one of the targeted fault conditions. The range of each fault condition, from the minimum required detection level to a maximum fault level, was defined. The complete fault range, from zero (nominal) to the maximum fault level, was divided arbitrarily into nine fault magnitude points. The SSME Power Balance Model was then used to generate simulated data for each of the 27 (3 targeted fault conditions for each of 9 distinct fault levels that included a nominal scenario) training case conditions. The results from all the simulations variables listed in table A1 were recorded. A matrix of the normalized simulation inputs and results were then used to generate a system diagnostic model. The development of the system diagnostic model, which is based on by an inverse model framework, is beyond the scope of this paper. However, detailed information regarding the inverse model is provided in the Systematic Sensor Selection Strategy (S4) User and Software Guide (ref. 34).

Once the system diagnostic model was established, simulation data at additional fault levels for the three fault cases were generated. The number and location of these testing cases should be selected to address diagnostic performance requirements properly. For this example, a single testing case was selected near the minimum required detection fault level for each fault scenario, in order to determine the impact of the selected sensor suite on the minimum detection requirements.

From each testing case, only the values for the sensors represented within the current suite being evaluated are utilized. An initial detection check is made to ensure that the current suite of values exhibits detectable values at a minimum level. If within the current sensor suite being evaluated, the defined detection criteria are not met, then the suite relative to this particular fault testing case is insensitive and the fault would be undetectable. For this demonstration, at least one measurement had to deviate from the nominal range by 3 times its sensor noise level or the combined root-mean-squared-error for the entire normalized sensor suite had to be greater than 0.25 , for the fault testing case to be detectable. If the sensor suite is determined to be insensitive, the diagnostic analysis is bypassed and another sensor suite is evaluated.

If the sensor suite is sensitive to the fault case, the sensor measurement values are passed through the system diagnostic model along with the nine training vectors for one of the possible fault cases. The system diagnostic model generates the recovered hardware variable representing the potential fault case in normalized form. Since the system diagnostic model uses an optimization process, there will not be an exact match of the input sensor values to the recovered sensor values. By computing this difference for each sensor value, the system diagnostic model also provides the residual sensor values of the recovered fault case. This process is repeated for each of the possible fault cases.

Post-processing of the system diagnostic model results provide performance parameters that can be used to determine the fault analysis ability of the current sensor suite. The S4 process allows the user to specify a wide range of metrics that are limited only in the imagination and programming ability of the user. The final performance metric for the sensor suite under evaluation in this example is a combination of a utility cost term, $U_{S}$, the diagnosability performance factor, $P_{F D}$ and user-specified penalty term, $P_{P E N}$. Each term derived for this example below.

$$
\text { Merit }=U_{S} * P_{\mathrm{FD}} * P_{\mathrm{PEN}}
$$

For this example, the recovered sensor values were compared to the actual input sensor values. The residuals beyond a normalized threshold (one standard deviation for all sensors in this example) were combined into a rootmean squared distance value for the selected sensor suite. 


$$
D_{\text {resid }}= \begin{cases}\sqrt{\sum_{i=0}^{N_{\mathrm{S}}}\left(\left|Y_{R_{i}}\right|-Y_{T_{i}}\right)^{2}} & \text { if }\left|Y_{R_{i}}\right|-Y_{T_{i}}>0 \\ 0 & \text { if }\left|Y_{R_{i}}\right|-Y_{T_{i}} \leq 0\end{cases}
$$

The distance value was computed for each testing fault scenario for each of the possible fault cases and combined into an overall metric for the sensor suite. The total distance value should reflect a preference for small residual distances (fault detection) when the fault input type matches the recovered fault type and large residuals (fault discrimination) when the fault types do not match. For each testing scenario,

$$
\begin{aligned}
D_{\text {Total }} & =\sum_{i=0}^{N_{\mathrm{FC}}}(-1)^{p} * D_{\text {resid } i} \\
\text { where } & \\
p & = \begin{cases}0 & \text { if input fault } \neq \text { fault }(i) \\
1 & \text { if input fault }=\text { fault }(i)\end{cases}
\end{aligned}
$$

The combined merit value is then a weighted linear combination of the total distance values for each of the tested fault scenarios. The weighting variables allow the user to prioritize the tested fault types by importance, occurrence, or criticality.

$$
P_{\mathrm{FD}}=\sum_{j=0}^{N_{\mathrm{FM}}} C_{j} * W_{j} * D_{\text {Total }_{j}}
$$

An additional metric is incorporated in the overall sensor suite performance value that specifies a utility cost term (weighting factor) for the sensor involved. This utility cost term could incorporate relative weighting values between the sensors, such as cost, weight, power requirements, et cetera, and is simply an average of pre-assigned values for each of the sensors involved in the evaluated suite.

$$
U_{S}=\frac{\sum_{i=0}^{N_{\mathrm{S}}} U_{i}}{N_{\mathrm{S}}}
$$

Finally, the penalty term is given by

$$
P_{\mathrm{PEN}}=\frac{K_{\mathrm{N}}}{K_{\mathrm{N}}+\left(W_{\text {penalty }} \times \mid \text { NSdesired }- \text { NSactual } \mid\right)}
$$

As established for this example, the penalty term includes two design parameters, $K_{\mathrm{N}}$ and $W_{\text {penalty }}$, which the user can specify initially. This term decreases from an optimal value of 1.0 as the number of sensors contained within the candidate suite deviates from the desired number of sensors that the user has specified.

\section{B. Results}

The S4 framework was executed with perturbations to parameters in the penalty term. The intent was to examine the number of actual sensors in the final solution sets versus the desired number of sensors selected by the user. The results are presented in table A2. The first column represents the desired number of sensors, the second column represents the weighting penalty applied and the remaining columns indicate the sensors selected from the S4 process. With $W_{\text {penalty }}$ values of 0.0 and 0.01 , the same three measurements, hpfpDp, hpotDT, and hpopIP, were selected regardless of the desired number of sensors specified. This measurement set scored well due to its 
sensitivity to the targeted fault conditions permitting it to overcome the penalty term as it was applied. When the $W_{\text {penalty }}$ value was raised to 0.1 , the penalty term was able to drive the solution set to the desired number of sensors with specific exceptions. When the target number of sensors was one, the minimal solution set contained opbPc and hpotDT. On the other extreme, as target sensors were increased to 9 and 10, opovX and opbFiP were never selected. Inclusion of these two measurements decreased the merit value more than the penalty term decreased the merit value for noninclusion.

Results from this example provide motivation for more investigation.

- As the target number of sensors increased, the same sensor set was selected again but with an additional sensor to satisfy the penalty term in the merit algorithm. This cannot always be expected when measurement information is combined in more complex examples.

- The ability exists within the established S4 framework to force the inclusion or exclusion of one or more sensors from the solution set. This feature could be used to examine the sensor set that would be selected if one or more of the primary three sensors (i.e., hpfpDp, hpotDT, and hpopIP) were not available.

- A more thorough analysis of the diagnostic performance results can be supported by the S4. This would provide design engineers the information of what system failures are covered and what failures still need to be addressed.

- A statistical evaluation can be performed on the potential sensor suite candidates to determine their sensitivity to system variations (e.g., signal noise and engine-to-engine build variations).

TABLE A2.-SELECTED SENSORS AS A FUNCTION OF THE DESIGNATED NUMBER OF SENSORS IN THE MEASUREMENT SUITE

\begin{tabular}{|c|c|c|c|c|c|c|c|c|c|c|c|}
\hline $\mathrm{N}_{\text {Sdesired }}$ & $W_{\text {penalty }}$ & hpfpDP & hpfpIP & IpftpS & hpopIP & hpotps & opbPc & opovX & mccOiP & opbFiP & hpotDT \\
\hline $\mathrm{n} / \mathrm{a}$ & 0 & $\bar{x}$ & & & & & $\bar{x}$ & & & & $\bar{X}$ \\
\hline 1 & 0.01 & $\mathrm{X}$ & & & & & $x$ & & & & $X$ \\
\hline 2 & 0.01 & $X$ & & & & & $X$ & & & & $X$ \\
\hline 3 & 0.01 & $\mathrm{X}$ & & & & & $\mathrm{X}$ & & & & $\mathrm{X}$ \\
\hline 4 & 0.01 & $\mathrm{X}$ & & & & & $\mathrm{X}$ & & & & $X$ \\
\hline 5 & 0.01 & $\mathrm{X}$ & & & & & $X$ & & & & $X$ \\
\hline 6 & 0.01 & $\mathrm{X}$ & & & & & $X$ & & & & $\mathrm{X}$ \\
\hline 7 & 0.01 & $\mathrm{X}$ & & & & & $\mathrm{X}$ & & & & $\mathrm{X}$ \\
\hline 8 & 0.01 & $X$ & & & & & $X$ & & & & $X$ \\
\hline 9 & 0.01 & $\mathrm{X}$ & & & & & $X$ & & & & $X$ \\
\hline 10 & 0.01 & $\bar{x}$ & & & & & $\mathrm{X}$ & & & & $\bar{X}$ \\
\hline 1 & 0.1 & & & & & & $\bar{x}$ & & & & $\mathrm{X}$ \\
\hline 2 & 0.1 & & & & & & $X$ & & & & $\mathrm{X}$ \\
\hline 3 & 0.1 & $X$ & & & & & $X$ & & & & $X$ \\
\hline 4 & 0.1 & $X$ & & & & $\mathrm{X}$ & $X$ & & & & $X$ \\
\hline 5 & 0.1 & $\mathrm{X}$ & & & & $\mathrm{X}$ & $\mathrm{X}$ & & $\mathrm{X}$ & & $\mathrm{X}$ \\
\hline 6 & 0.1 & $X$ & & $\mathrm{X}$ & & $\mathrm{X}$ & $X$ & & $\mathrm{X}$ & & $\mathrm{X}$ \\
\hline 7 & 0.1 & $\mathrm{X}$ & & $\mathrm{X}$ & $\mathrm{X}$ & $\mathrm{X}$ & $x$ & & $\mathrm{X}$ & & $\mathrm{X}$ \\
\hline 8 & 0.1 & $X$ & $X$ & $X$ & $\mathrm{X}$ & $X$ & $X$ & & $\mathrm{X}$ & & $X$ \\
\hline 9 & 0.1 & $\mathrm{X}$ & $\mathrm{X}$ & $\mathrm{X}$ & $X$ & $\mathrm{X}$ & $\mathrm{X}$ & & $\mathrm{X}$ & & $X$ \\
\hline 10 & 0.1 & $\mathrm{X}$ & $\mathrm{X}$ & $\mathrm{X}$ & $X$ & $\mathrm{X}$ & $\mathrm{X}$ & & $X$ & & 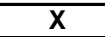 \\
\hline
\end{tabular}

\section{References}

1. Yoo, T.-S., and Lafortune, S., "NP-Completeness of Sensor Selection Problems Arising in Partially Observed Discrete-Event Systems,” IEEE Transactions on Automatic Control, vol. 47, 2002, pp. 1495-1499.

2. Debouk, R., Lafortune, S., and Teneketzis, D., "On an Optimization Problem in Sensor Selection for Failure Diagnosis," Proceedings of the 38th Conference on Decision \& Control, vol. 5, IEEE, 1999, pp. 4990-4995.

3. Worden, K., and Burrows, A., "Optimal Sensor Placement for Fault Detection,” Engineering Structures, vol. 23, 2001, pp. 885-901.

4. Eberhart, R., and Kennedy, J., “A New Optimizer Using Particle Swarm Theory,” IEEE Sixth International Symposium on Micro Machine and Human Science, IEEE, 1995, pp. 39-43.

5. Osorio, M., and Hernández, E., "Cutting Analysis for MKP," IEEE Proceedings of the Fifth Mexican International Conference in Computer Science, ENC’04, IEEE, 2004, pp. 298-303.

6. Fletcher, R., Practical Methods of Optimization, 2nd ed., John Wiley \& Sons, Chichester, 1987. 
7. Narasimhan, S., Mosterman, P., and Biswas, G., "A Systematic Analysis of Measurement Selection Algorithms for Fault Isolation in Dynamic Systems," Proceedings of the 9th International Workshop on Principles of Diagnosis, DX-98, 1998, pp. 94-101.

8. Mushini, R., and Simon, D., "On Optimization of Sensor Selection for Aircraft Gas Turbine Engines," Proceedings of the 18th International Conference on Systems Engineering, ISCEng'05, IEEE, 2005, pp. 9-14.

9. Madron, F., and Veverka, V., "Optimal Selection of Measuring Point in Complex Plants by Linear Models," American Institute of Chemical Engineering Journal, vol. 38, no. 2, 1992, pp. 227-236.

10. Musulin, E., Benqlilou, C., Bagajewicz, M., and Puigjaner, L., "Instrumentation Design Based on Optimal Kalman Filtering,” Journal of Process Control, vol. 15, no. 6, 2005, pp. 629-638.

11. Spanache, S., Escobet, T., and Travé-Massuyès, L., "Sensor Placement Optimisation Using Genetic Algorithms," Proceedings of the15th International Workshop on Principles of Diagnosis, DX-04, 2004, pp. 179-183.

12. Sen S., S. Narasimhan and K. Deb, "Sensor Network Design of Linear Processes Using Genetic Algorithms," Computers and Chemical Engineering, 1998, vol. 22, 1998, pp. 385-390.

13. Santi, L.M, Sowers, T.S. and Aguilar, R.B., "Optimal Sensor Selection for Health Monitoring Systems," NASA/TM-2005-213955, 2005.

14. Zhang, G., “Optimum Sensor Localization/Selection in a Diagnostic/Prognostic Architecture,” Ph.D. Dissertation, Electrical and Computer Engineering Dept., Georgia Institute of Technology, 2005.

15. Ali, Y., and Narasimhan, S., "Sensor Network Design for Maximizing Reliability of Linear Processes," American Institute of Chemical Engineering Journal, vol. 39, no. 5, 1993, pp. 820-828.

16. Ali, Y., and Narasimhan, S., "Redundant Sensor Network Design for Linear Processes," American Institute of Chemical Engineering Journal, vol. 41, no. 10, 1995, pp. 2237-2249.

17. Bagajewicz, M., "Design and Retrofit of Sensor Networks in Process Plants," American Institute of Chemical Engineering Journal, vol. 43, no. 9, 1997, pp. 2300-2306.

18. Bagajewicz, M., and Sanchez, M., "Design and Upgrade of Nonredundant and Redundant Linear Sensor Networks," American Institute of Chemical Engineering Journal, vol. 45, no. 9, 1999, pp. 1927-1938.

19. Azam, M., Pattipati, K., and Patterson-Hine, A., "Optimal Sensor Allocation for Fault Detection and Isolation," IEEE International Conference on Systems, Man and Cybernetics, vol. 2, IEEE, 2004, pp. 1309-1314.

20. Bagajewicz, M., and Sanchez, M., "Cost-optimal Design of Reliable Sensor Networks," Computers and Chemical Engineering Journal, vol. 23, 2000, pp. 1757-1762.

21. Bagajewicz, M., and Cabrera, E., "New MILP Formulation for Instrumentation Network Design and Upgrade," American Institute of Chemical Engineering Journal, vol. 48, no. 10, 2002, pp. 2271-2282.

22. Bagajewicz, M., Fuxman, A., and Uribe, A., "Instrumentation Network Design and Upgrade for Process Monitoring and Fault Detection,” American Institute of Chemical Engineering Journal, vol. 50, no. 8, 2004, pp. 1870-1880.

23. Chmielewski, D., Palmer, T., and Manousiouthakis, V., "On the Theory of Optimal Sensor Placement," American Institute of Chemical Engineering Journal, vol. 48, no. 5, 2002, pp. 1001-1012.

24. Kretsovalis, A., and Mah, R., "Observability and Redundancy Classification in Multicomponent Process Networks," American Institute of Chemical Engineering Journal, vol. 33, no. 1, 1987, pp. 70-82.

25. Luong, M., Maquin, D., Huynh C., and Ragot, J., "Observability, Redundancy, Reliability and Integrated Design of Measurement System," Proceedings of 2nd IFAC Symposium on Intelligent Components and Instruments for Control Applications, SICICA'94, 1994.

26. Dochain, D., Tali-Maanar, N., and Babary, J.P., "On Modeling, Monitoring and Control of Fixed Bed Bioreactors,” Computers and Chemical Engineering, vol. 21, 1997, pp. 1255-1266.

27. Muller, P., and Weber, H., "Analysis and Optimization of Certain Qualities of Controllability and Observability for Linear Dynamical Systems,” Automatica, vol. 8, 1972, pp. 237-246.

28. Van den Berg, F., Hoefsloot, H., Boelens, H., and Smilde, A., "Selection of Optimal Sensor Position in a Tubular Reactor Using Robust Degree of Observability Criteria,” Chemical Engineering Science, 2000, vol. 55, 2000, pp. 827-837.

29. Papadopoulos, M., and Garcia, E., "Sensor Placement Methodologies for Dynamic Testing," AIAA Journal, vol. 36, no. 2, 1998, pp. 256-263.

30. Hac, A., and Liu, L., "Sensor and Actuator Location in Motion Control of Flexible Structures," Journal of Sound and Vibration, vol. 167, no. 2, 1993, pp. 239-261.

31. Raghuraj, R., Bhushan, M., and Rengaswamy, R., "Locating Sensors in Complex Chemical Plants Based on Fault Diagnostic Observability Criteria,” American Institute of Chemical Engineering Journal, vol. 45, no. 2, 1999, pp. 310-322. 
32. Bhushan, M., and Rengaswamy, R., "Design of Sensor Network Based on the Signed Directed Graph of the Process for Efficient Fault Diagnosis,” Industrial and Engineering Chemistry Research, vol. 39, 2000, pp. 9991019.

33. Yan, Y., "Sensor Placement and Diagnosability Analysis at Design Stage,” National Research Council of Canada, NRC 47160, 2004.

34. Sowers, T.S., “Systematic Sensor Selection Strategy User and Software Guide,” NASA TM, (to be published). 


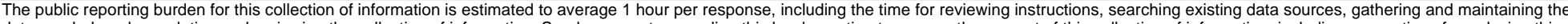

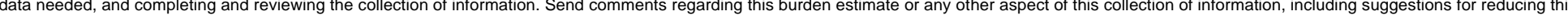

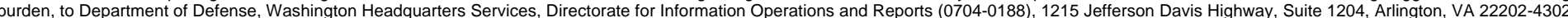

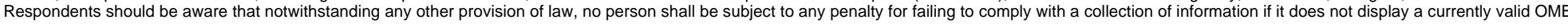
control number.

PLEASE DO NOT RETURN YOUR FORM TO THE ABOVE ADDRESS

\section{REPORT DATE (DD-MM- $Y Y Y Y)$ \\ 2. REPORT TYPE \\ 3. DATES COVERED (From - To)}

16-07-2007

\section{TITLE AND SUBTITLE}

Technical Memorandum

Sensor Selection and Optimization for Health Assessment of Aerospace Systems

6. AUTHOR(S)

Maul, William, A.; Kopasakis, George; Santi, Louis, M.; Sowers, Thomas, S.; Chicatelli, Amy

5a. CONTRACT NUMBER

5b. GRANT NUMBER

5c. PROGRAM ELEMENT NUMBER

\section{5d. PROJECT NUMBER}

5e. TASK NUMBER

5f. WORK UNIT NUMBER

WBS 136905.08.05.08.08.01.03

\section{PERFORMING ORGANIZATION NAME(S) AND ADDRESS(ES)}

National Aeronautics and Space Administration

8. PERFORMING ORGANIZATION

REPORT NUMBER

John H. Glenn Research Center at Lewis Field

E-15992

Cleveland, Ohio 44135-3191

\section{SPONSORING/MONITORING AGENCY NAME(S) AND ADDRESS(ES)}

National Aeronautics and Space Administration

Washington, DC 20546-0001

$\begin{aligned} & \text { 10. SPONSORING/MONITORS } \\ & \text { ACRONYM(S) } \\ & \text { NASA }\end{aligned}$
$\begin{aligned} & \text { 11. SPONSORING/MONITORING } \\ & \text { REPORT NUMBER } \\ & \text { NASA/TM-2007-214822; AIAA-2007- } \\ & 2849\end{aligned}$

\section{DISTRIBUTIONIAVAILABILITY STATEMENT}

Unclassified-Unlimited

Subject Categories: 06 and 19

Available electronically at http://gltrs.grc.nasa.gov

This publication is available from the NASA Center for AeroSpace Information, 301-621-0390

\section{SUPPLEMENTARY NOTES}

\section{ABSTRACT}

Aerospace systems are developed similarly to other large-scale systems through a series of reviews, where designs are modified as system requirements are refined. For space-based systems few are built and placed into service-these research vehicles have limited historical experience to draw from and formidable reliability and safety requirements, due to the remote and severe environment of space. Aeronautical systems have similar reliability and safety requirements, and while these systems may have historical information to access, commercial and military systems require longevity under a range of operational conditions and applied loads. Historically, the design of aerospace systems, particularly the selection of sensors, is based on the requirements for control and performance rather than on health assessment needs. Furthermore, the safety and reliability requirements are met through sensor suite augmentation in an ad hoc, heuristic manner, rather than any systematic approach. A review of the current sensor selection practice within and outside of the aerospace community was conducted and a sensor selection architecture is proposed that will provide a justifiable, defendable sensor suite to address system health assessment requirements.

\section{SUBJECT TERMS}

Sensor selection; Sensor placement; Diagnostics; Health assessment

\begin{tabular}{|c|c|c|c|}
\hline \multicolumn{3}{|c|}{ 16. SECURITY CLASSIFICATION OF: } & \multirow{2}{*}{$\begin{array}{l}\text { 17. LIMITATION OF } \\
\text { ABSTRACT }\end{array}$} \\
\hline $\begin{array}{l}\text { a. REPORT } \\
\text { U }\end{array}$ & $\begin{array}{l}\text { b. ABSTRACT } \\
\text { U }\end{array}$ & $\begin{array}{l}\text { c. THIS } \\
\text { PAGE } \\
\text { U }\end{array}$ & \\
\hline
\end{tabular}

18. NUMBER
OF
PAGES
21

19a. NAME OF RESPONSIBLE PERSON
William A. Maul
19b. TELEPHONE NUMBER (include area code)
216-977-7496

Standard Form 298 (Rev. 8-98)

Prescribed by ANSI Std. Z39-18 

